\title{
Application of Convex Functions and Jensen Inequality
}

\author{
Hua Zhong ${ }^{\mathrm{a}}$, Xuemei Yang ${ }^{\mathrm{b}}$, WuSheng Wang ${ }^{\mathrm{c}}$ \\ School of Mathematics and Statistics, Hechi University, Guangxi, Yizhou 546300, P. R. China \\ aasonfan@163.com, b1160455368@qq.com, cwang4896@126.com
}

Keywords: Convex function; Jensen inequality; Integral inequality; Application.

\begin{abstract}
Functions are the important fundamental concepts in mathematics and the key research object in mathematical analysis. Convex function is a class of very important functions which is widely used in pure mathematics, functional analysis, optimization theory, mathematical economics. This paper firstly introduces a definition of convex function, and then gives properties of convex function and its judging theorem, finally, gives examples which show how to prove more complex inequalities using Jensen inequality of discrete form.
\end{abstract}

\section{Introduction}

Since the beginning of twenty-first Century the establishment of the theory of convex functions, the concept of convex function has been widely used in many branches of mathematics, for example in mathematical analysis, function theory, functional analysis, optimization theory and so on. Many authors [1-11] studied properties and applications of convex function.

Definition (see [1]) Let $f(x)$ be a function defined on interval $\boldsymbol{I}$. If

$$
f\left(\lambda x_{1}+(1-\lambda) x_{2}\right) \leq \lambda f\left(x_{1}\right)+(1-\lambda) f\left(x_{2}\right), \forall x_{1}, x_{2} \in I, \lambda \in(0,1) .
$$

Then, $f(x)$ is called a convex function on interval $\boldsymbol{I}$, and $f(x)$ is called a strict convex function on interval I when the inequality (1) is the strict inequality.

Lemma 1 (see [2]) Suppose that $f(x)$ is a continuous function defined on the closed interval $[a, b]$, and is differential on the open interval $(a, b)$. Then $f(x)$ is a convex function on the closed interval $[a, b]$ when and only when

$f^{\prime \prime}(x) \geq 0, x \in(a, b)$.

$f(x)$ is a strict convex function when and only when

$f^{\prime \prime}(x)>0, x \in(a, b)$.

Lemma 2 (see [3]) (Jensen inequality of discrete form) Suppose that $f(x)$ is a convex function on interval $[a, b]$. Then, for any $\forall x_{i} \in[a, b], i=1,2, \cdots, n, \lambda_{i} \in(0,1), \sum_{i=1}^{n} \lambda_{i}=1$, the inequality

$$
f\left(\sum_{i=1}^{n} \lambda_{i} x_{i}\right) \leq \sum_{i=1}^{n} \lambda_{i} f\left(x_{i}\right)
$$

holds.

This paper discusses how to prove more complex inequalities using Jensen inequality of discrete form.

\section{Application of Jensen inequality of discrete form}

In many problems, we often encountered in the proof of some inequalities. Proof of inequality often requires some high skills. Proof process can be made to be simple and clear using Jensen inequality of the discrete form of a convex function. But the key of this method is constructing appropriate convex functions

Example 1 (see [4]) Prove the inequality 
$\frac{e^{x}+e^{y}}{2}>e^{\frac{x+y}{2}}$

Proof: Let $f(t)=e^{t}$, we have $f^{\prime \prime}(t)=e^{t}>0$, then

$f(t)$ is a strict convex function on interval $(-\infty,+\infty)$. From (1), we have

$\frac{e^{x}+e^{y}}{2}>e^{\frac{x+y}{2}}$.

Example 2(see [1]) Prove the inequality

$(a b c)^{\frac{a+b+c}{3}}<a^{a} b^{b} c^{c}$.

where $a, b, c$ are positive real numbers.

Proof: Let $f(x)=x \ln x, x>0$, then

$$
f^{\prime}(x)=1+\ln x, f^{\prime \prime}(x)=\frac{1}{x}>0 .
$$

By Lemma 1 , we see that $f(x)$ is a convex function on interval $(0,+\infty)$. From (4)in Lemma 2, we have

$$
f\left(\frac{a+b+c}{3}\right)<\frac{1}{3}(f(a)+f(b)+f(c))
$$

i.e.

$$
\frac{a+b+c}{3} \ln \frac{a+b+c}{3}<\frac{1}{3}(a \ln +b \ln b+c \ln c) .
$$

It implies that $\left(\frac{a+b+c}{3}\right)^{a+b+c}<a^{a} b^{b} c^{c}$.

Since $\sqrt[3]{a b c}<\frac{a+b+c}{3}$, we have

$$
(a b c)^{\frac{a+b+c}{3}}<a^{a} b^{b} c^{c} \text {. }
$$

Example 3(see [5]) Prove the following inequalities

(a) $\arctan \left(\lambda_{1} a_{1}+\cdots+\lambda_{n} a_{n}\right) \geq \lambda_{1} \arctan a_{1}+\cdots+\lambda_{n} \arctan a_{n}$;

(b) $\lambda_{1} a_{1}+\lambda_{2} a_{2}+\cdots+\lambda_{n} a_{n} \geq a_{1}^{\lambda_{1}} \cdot a_{2}^{\lambda_{2}} \cdots \cdots a_{n}^{\lambda_{n}}$.

where $a_{j} \geq 0, \lambda_{j} \geq 0, j=1,2, \cdots, n, \lambda_{1}+\lambda_{2}+\cdots+\lambda_{n}=1$.

Proof: (a) Let $f(x)=-\arctan x, x \in(0,+\infty)$, then

$$
f^{\prime}(x)=-\frac{1}{1+x^{2}}, f^{\prime \prime}(x)=\frac{2 x}{\left(1+x^{2}\right)^{2}}>0, x \in(0,+\infty) \text {. }
$$

Using Lemma 1 , we see that $f(x)$ is a strict convex function on interval $(0,+\infty)$. From Jensen inequality (4), we get

$-\arctan \left(\lambda_{1} a_{1}+\cdots+\lambda_{n} a_{n}\right) \leq-\left(\lambda_{1} \arctan a_{1}+\cdots+\lambda_{n} \arctan a_{n}\right)$.

It implies the required inequality $\arctan \left(\lambda_{1} a_{1}+\cdots+\lambda_{n} a_{n}\right) \geq \lambda_{1} \arctan a_{1}+\cdots+\lambda_{n} \arctan a_{n}$.

(b)Let $f(x)=-\ln x, x \in(0,+\infty)$, then

$$
f^{\prime}(x)=-\frac{1}{x}, f^{\prime \prime}(x)=\frac{1}{x_{2}}>0, x \in(0,+\infty) .
$$

We see that $f(x)$ is a strict convex function on interval $(0,+\infty)$. By Jensen inequality (4), we obtain $-\ln \left(\lambda_{1} a_{1}+\lambda_{2} a_{2}+\cdots+\lambda_{n} a_{n}\right) \leq-\left(\lambda_{1} \ln a_{1}+\lambda_{2} \ln a_{2}+\cdots+\lambda_{n} \ln a_{n}\right)=-\ln \left(a_{1}^{\lambda_{1}} \cdot a_{2}^{\lambda_{2}} \cdots \cdots a_{n}^{\lambda_{n}}\right)$, i.e. $\lambda_{1} a_{1}+\lambda_{2} a_{2}+\cdots+\lambda_{n} a_{n} \geq a_{1}^{\lambda_{1}} \cdot a_{2}^{\lambda_{2}} \cdots \cdots a_{n}^{\lambda_{n}}$. 
Example 4(see [6]) Suppose that $a_{i}, b_{i} \in R^{+}(i=1,2, \cdots, n), \sum_{i=1}^{n} a_{i}=\sum_{i=1}^{n} b_{i}$, then

$$
\sum_{i=1}^{n} \frac{a_{i}^{2}}{a_{i}+b_{i}} \geq \frac{1}{2} \sum_{i=1}^{n} a_{i}
$$

Proof: Let $S=\sum_{i=1}^{n} a_{i}$, then $S=\sum_{i=1}^{n} b_{i}, \sum_{i=1}^{n} \frac{a_{i}}{S}=1$. Let $f(x)=\frac{1}{1+x}, x \in(0, \infty)$,

then $f^{\prime}(x)=-\frac{1}{(1+x)^{2}}, f^{\prime \prime}(x)=\frac{2}{(1+x)^{3}}>0, x \in(0, \infty)$. So $f(x)$ is a strict convex function on interval $(0,+\infty)$. Let $x_{i}=\frac{b_{i}}{a_{i}}, i=1,2, \cdots, n$. Since $\sum_{i=1}^{n} a_{i}=\sum_{i=1}^{n} b_{i}=S$, we have

$$
\begin{aligned}
\sum_{i=1}^{n} \frac{a_{i}^{2}}{a_{i}+b_{i}} & =S \sum_{i=1}^{n} \frac{a_{i}}{S} \cdot \frac{1}{1+x_{i}}=S \sum_{i=1}^{n} \frac{a_{i}}{S} f\left(x_{i}\right)>S f\left(\sum_{i=1}^{n} \frac{a_{i}}{S} x_{i}\right) \\
& =S \frac{1}{1+\sum_{i=1}^{n} \frac{a_{i}}{S} x_{i}}=\frac{S}{1+\sum_{i=1}^{n} \frac{b_{i}}{S}}=\frac{S}{2}=\frac{1}{2} \sum_{i=1}^{n} a_{i},
\end{aligned}
$$

i.e.

$$
\sum_{i=1}^{n} \frac{a_{i}^{2}}{a_{i}+b_{i}} \geq \frac{1}{2} \sum_{i=1}^{n} a_{i}
$$

Example 5(see [7]) Suppose that $g(x)$ is a integrable function on interval[ $a, b]$ with $c \leq g(x) \leq d, f(y)$ is a convex function on interval $[c, d]$, then

$$
f\left(\frac{1}{b-a} \int_{a}^{b} g(x) d x\right) \leq \frac{1}{b-a} \int_{a}^{b} f[g(x)] d x .
$$

Proof: Since $f(y)$ (or $g(x))$ is integrable function on interval $[c, d]$ (or on interval $[a, b]$ ), we have

$f\left(\frac{1}{b-a} \lim _{n \rightarrow \infty} \sum_{i=1}^{n} g\left(a+\frac{b-a}{n} i\right) \frac{b-a}{n}\right)=f\left(\frac{1}{b-a} \int_{a}^{b} g(x) d x\right)$,

$\frac{1}{b-a} \lim _{n \rightarrow \infty} \sum_{i=1}^{n} f\left(g\left(a+\frac{b-a}{n} i\right)\right) \frac{b-a}{n}=\frac{1}{b-a} \int_{a}^{b} f[g(x)] d x$.

Let $y_{i}=g\left(a+\frac{b-a}{n} i\right), i=1,2, \cdots n$. Since $f(y)$ is a convex function on interval $[c, d]$, using Jensen inequality

(4), we have

$$
\begin{aligned}
& f\left(\frac{1}{b-a} \sum_{i=1}^{n} g\left(a+\frac{b-a}{n} i\right) \frac{b-a}{n}\right)=f\left(\frac{1}{n} \sum_{i=1}^{n} y_{i}\right) \leq \frac{1}{n} \sum_{i=1}^{n} f\left(y_{i}\right) \\
& =\frac{1}{b-a} \sum_{i=1}^{n} f\left(g\left(a+\frac{b-a}{n} i\right)\right) \frac{b-a}{n} .
\end{aligned}
$$

Limiting on both sides of the above inequality, we have

$$
\lim _{n \rightarrow \infty} f\left(\frac{1}{b-a} \sum_{i=1}^{n} g\left(a+\frac{b-a}{n} i\right) \frac{b-a}{n}\right) \leq \lim _{n \rightarrow \infty} \frac{1}{b-a} \sum_{i=1}^{n} f\left(g\left(a+\frac{b-a}{n} i\right)\right) \frac{b-a}{n} .
$$

Since $f(y)$ is a continuous function, we obtain

$$
f\left(\frac{1}{b-a} \lim _{n \rightarrow \infty} \sum_{i=1}^{n} g\left(a+\frac{b-a}{n} i\right) \frac{b-a}{n}\right) \leq \frac{1}{b-a} \lim _{n \rightarrow \infty} \sum_{i=1}^{n} f\left(g\left(a+\frac{b-a}{n} i\right)\right) \frac{b-a}{n} .
$$


By the Integrable property of $f$ and $f \circ g$, we have proved the required inequality

$f\left(\frac{1}{b-a} \int_{a}^{b} g(x) d x\right) \leq \frac{1}{b-a} \int_{a}^{b} f[g(x)] d x$.

\section{Summary}

In many problems, we often encountered in the proof of some inequalities. Proof of inequality often requires some high skills. Proof process can be made to be simple and clear using Jensen inequality of the discrete form of a convex function. But the key of this method is constructing appropriate convex functions. This paper gives examples which show how to prove more complex inequalities using Jensen inequality of discrete form.

\section{Acknowledgement}

This research was supported by Guangxi Natural Science Foundation (Project No. 2012GXNSFAA053009) and Scientific Research Foundation of the Education Department of Guangxi Autonomous Region of China (No. KY2015ZD103, KY2015LX340).

\section{References}

[1] Department of mathematics, East China Normal University, Mathematical analysis, third ed., Higher Education Press, Beijing, 2001.

[2] M. Zhou, Drilling of mathematical analysis exercises, Science Press, Beijing, 2006.

[3] J. Kuang, Common inequality Fourth Edition, Shandong science and Technology Press, Jinan, 2010.

[4] L.Yu, X. Zhao and D. Song, Advanced mathematics theory and solving method, Chemical Industry Press, Beijing,2010.

[5] L.Wu, Y. Mao, G. Song and M. Wei, Solutions precision of exercises of mathematical analysis, Science Press, Beijing, 2002.

[6] R. Li, Proving inequalities using convex function, Journal of Ningde Teachers College(Natural Science), 10(1)(1998)74-75.

[7] X. Cui and L. Liu, Jensen inequality and its applications, Journal of Handan Teachers College, 11(3)(2001)23-26.

[8] M. Alomari and M. Darus, The Hadamard's inequality for s-convex function of 2-variables On The co-ordinates, Int. Journal of Math. Analysis. 2 (13) (2008) 629-638.

[9] B. Xi, F. Qi, Some inequalities of Hermite-Hadamard type for h-convex functions, Adv. Inequal. Appl. 2 (1) (2013) 1-15.

[10] S.S. Dragomir, S. Fitzpatrik, The Hadamard's inequality for s-convex functions in the second sense, Demonstration Math. 32 (4) (1999) 687-696.

[11] M.Z. Sarikaya, A. Saglam, H. Yildirim, On some Hadamard-type inequalities for h-convex functions, J. Math. Inequalities. 2 (2008) 335-341. 